\title{
Kummer congruences for values of Bernoulli and Euler polynomials
}

\author{
by \\ Paul Thomas Young (Charleston, SC)
}

Dedicated to the memory of Bernard Dwork

1. Introduction. The Bernoulli polynomials $B_{n}(x)$ may be defined by

$$
\left(\frac{t}{e^{t}-1}\right) e^{x t}=\sum_{n=0}^{\infty} B_{n}(x) \frac{t^{n}}{n !},
$$

and their values at $x=0$ are called the Bernoulli numbers and denoted $B_{n}$. The strong form of the Kummer congruences (cf. [1]) states that if $p$ is an odd prime, $c \equiv 0\left(\bmod (p-1) p^{a}\right)$, and $p-1$ does not divide $m$, then

$$
\Delta_{c}^{k}\left\{\left(1-p^{m-1}\right) \frac{B_{m}}{m}\right\} \equiv 0\left(\bmod p^{k(a+1)} \mathbb{Z}_{p}\right),
$$

where $\Delta_{c}$ is the forward difference operator with increment $c$ and $\Delta_{c}^{k}$ denotes the $k$ th compositional iterate of this operator.

In this paper we give a generalization to Bernoulli polynomials $B_{n}(x)$ where the argument $x$ may be any $p$-adic integer. Specifically, we show in Theorem 3.2 below that

$$
\Delta_{c}^{k}\left\{\frac{B_{m}(x)-p^{m-1} B_{m}\left(x^{\prime}\right)}{m}\right\} \equiv 0\left(\bmod p^{k(a+1)} \mathbb{Z}_{p}\right)
$$

under the above hypotheses, as well as an extension to the case $p=2$. The map $x \mapsto x^{\prime}$ appearing in (1.3) is Dwork's shift map, defined for $x \in \mathbb{Z}_{p}$ by the relation $p x^{\prime}-x=\mu_{x} \in\{0,1, \ldots, p-1\}$ (cf. [3], Ch. 8). A version of this result was first given in [4] in the case where $k=1, p \geq 5$, and $x \in \mathbb{Z}_{p} \cap \mathbb{Q}$. Our method is based on general properties of the $p$-adic $\Gamma$ transform recorded in [11] and yields a nontrivial analogous result in the case $p=2$. We also treat the Euler polynomials $H_{n}(u, x)$ in the same manner.

2000 Mathematics Subject Classification: 11B68, 11S40, 11S80. 
Additionally, in each case we give an analogous congruence with $\Delta_{c}^{k}$ replaced by a binomial coefficient operator, as in [7].

In $\S 4$ we prove Kummer congruences for generalized Bernoulli polynomials $B_{n, \chi}(x)$ associated to a Dirichlet character $\chi$. We demonstrate in Theorem 4.2 an analogue of (1.3) for $B_{m, \chi \omega^{-m}}(x)$ which holds for $x \in p \mathbb{Z}_{p}$ as long as $\chi$ is not a character of the second kind. The members of these congruences coincide with values of the two-variable $p$-adic $L$ function defined by G. Fox in [6]. We also show how this congruence can be extended in certain cases where $\chi$ is a character of the first kind and $x$ lies in $\mathbb{Z}_{p}^{\times}$.

2. Preliminaries. Throughout this paper $p$ will denote a prime number, $\mathbb{Z}_{p}$ the ring of $p$-adic integers, and $\mathbb{Q}_{p}$ the field of $p$-adic numbers. If $K$ is a finite extension of $\mathbb{Q}_{p}$ then $\mathfrak{O}_{K}$ will denote its ring of integers and $\mathfrak{O}_{K}^{\times}$ will denote the multiplicative group of units in $\mathfrak{O}_{K}$. Define the quantity $q$ by setting $q=p$ if $p>2$ and $q=4$ if $p=2$. The Teichmüller character $\omega$ on $\mathbb{Z}_{p}^{\times}$ is defined by setting $\omega(x)$ to be the unique $\phi(q)$ th root of unity congruent to $x$ modulo $q \mathbb{Z}_{p}$. We use $\mathfrak{O}_{K}[T-1]$ and $\mathfrak{O}_{K}[[T-1]]$ to denote respectively the ring of polynomials and of formal power series in the indeterminate $T-1$ over $\mathfrak{O}_{K}$. We use "ord $p$ " to denote the additive valuation on $K$ normalized by $\operatorname{ord}_{p} p=1$. Finally, $e^{t}$ denotes the exponential function defined by the power series $\sum_{n=0}^{\infty} t^{n} / n$ ! for $\operatorname{ord}_{p} t>1 /(p-1)$.

If $c$ is a nonnegative integer, the difference operator $\Delta_{c}$ operates on the sequence $\left\{a_{m}\right\}$ by

$$
\Delta_{c} a_{m}=a_{m+c}-a_{m} .
$$

The powers $\Delta_{c}^{k}$ of $\Delta_{c}$ are defined by $\Delta_{c}^{0}=$ identity and $\Delta_{c}^{k}=\Delta_{c} \circ \Delta_{c}^{k-1}$ for positive integers $k$, so that

$$
\Delta_{c}^{k} a_{m}=\sum_{j=0}^{k}\left(\begin{array}{l}
k \\
j
\end{array}\right)(-1)^{k-j} a_{m+j c}
$$

for all nonnegative integers $k$. To define binomial coefficient operators $\left(\begin{array}{l}D \\ k\end{array}\right)$ associated to an operator $D$ (cf. [7]), we write the binomial coefficient

$$
\left(\begin{array}{l}
X \\
k
\end{array}\right)=\frac{X(X-1) \ldots(X-k+1)}{k !}
$$

for $k \geq 0$ as a polynomial in $X$, and replace $X$ by $D$. Since the particular sequences considered in this paper have multiple indices, we shall always use the index $m$ to denote the index on which an operator operates.

Define the linear operator $\varphi$ by

$$
\varphi h(T)=h(T)-\frac{1}{p} \sum_{\zeta^{p}=1} h(\zeta T) .
$$


This operator is well defined and stable on rational functions, and also on $\mathfrak{O}_{K}[[T-1]]$ (cf. [11], (2.14)). If $h\left(e^{t}\right)=\sum a_{n} t^{n} / n$ !, write $(\varphi h)\left(e^{t}\right)=$ $\sum \widehat{a}_{n} t^{n} / n$ !. The following congruences for the numbers $\widehat{a}_{n}$ were proved in [11]:

TheOREM 2.1. Let $h \in \mathfrak{O}_{K}[[T-1]]$ and write $\varphi h\left(e^{t}\right)=\sum_{n=0}^{\infty} \widehat{a}_{n} t^{n} / n$ !. Then $\widehat{a}_{n} \in \mathfrak{O}_{K}$ for all $n$. Furthermore, if $c \equiv 0\left(\bmod \phi(q) p^{a}\right)$ with $a \geq 0$ then

$$
\Delta_{c}^{k} \widehat{a}_{m} \equiv 0\left(\bmod p^{k a^{+}} \mathfrak{O}_{K}\right)
$$

for all $m, k \geq 0$, where $a^{+}=a+1$ if $p>2$ and $a^{+}=a+3$ if $p=2$, and also

$$
\left(\begin{array}{c}
p^{-r} \Delta_{c} \\
k
\end{array}\right) \widehat{a}_{m} \in \mathfrak{O}_{K}
$$

for $0 \leq r \leq a^{+}$and all $m, k \geq 0$.

It will be observed from this theorem that the operator $\left(p^{-a^{+}} \Delta_{c}\right)^{k}$ is a polynomial of order $k$ in $\Delta_{c}$ with leading coefficient $p^{-k a^{+}}$which sends $\widehat{a}_{m}$

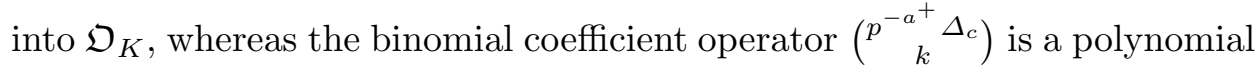
of order $k$ in $\Delta_{c}$ with leading coefficient $p^{-k a^{+}} / k$ ! which sends $\widehat{a}_{m}$ into $\mathfrak{O}_{K}$.

The proof of this theorem made use of the correspondence

$$
\Lambda \leftrightarrow \mathfrak{O}_{K}[[T-1]]
$$

where $\Lambda$ denotes the set of all $\mathfrak{O}_{K}$-valued measures on $\mathbb{Z}_{p}$, under which each measure $\alpha \in \Lambda$ corresponds to the formal power series $h \in \mathfrak{O}_{K}[[T-1]]$ defined by

$$
h(T)=\int_{\mathbb{Z}_{p}} T^{x} d \alpha(x) .
$$

From this it follows that

$$
a_{n}=\int_{\mathbb{Z}_{p}} x^{n} d \alpha(x) .
$$

We also observed $([11],(2.14))$ that

$$
\varphi h(T)=\int_{\mathbb{Z}_{p}^{\times}} T^{x} d \alpha(x),
$$

which implies

$$
\widehat{a}_{n}=\int_{\mathbb{Z}_{p}^{\times}} x^{n} d \alpha(x) .
$$

Since

$$
(1-\varphi) h(T)=\int_{p \mathbb{Z}_{p}} T^{x} d \alpha(x)=\int_{\mathbb{Z}_{p}} T^{p x} d \alpha(p x),
$$

we see that $(1-\varphi) h(T) \in \mathfrak{O}_{K}\left[\left[T^{p}-1\right]\right]$ according to the correspondence 
(2.5), (2.6). Therefore there is a linear operator $\psi$ on $\mathfrak{O}_{K}[[T-1]]$ such that $(\psi h)\left(T^{p}\right)=(1-\varphi) h(T)$. From $(2.4)$ we see that the operator $\psi$ may be defined by

$$
\psi h(T)=\frac{1}{p} \sum_{Z^{p}=T} h(Z),
$$

and therefore coincides on $\mathfrak{O}_{K}[[T-1]]$ with Dwork's $\psi$ operator (cf. [3], Ch. 5). So if we write $(\psi h)\left(e^{t}\right)=\sum_{n=0}^{\infty} a_{n}^{*} t^{n} / n$ !, then $a_{n}^{*} \in \mathfrak{O}_{K}$ for all $n$ and

$$
\widehat{a}_{n}=a_{n}-p^{n} a_{n}^{*} \text {. }
$$

Now if $g \in \mathfrak{O}_{K}[[T-1]]$ and $g\left(e^{t}\right)=\sum_{n=0}^{\infty} u_{n} t^{n} / n$ !, let us define polynomials $u_{n}(x) \in \mathfrak{O}_{K}[x]$ by

$$
e^{x t} g\left(e^{t}\right)=\sum_{n=0}^{\infty} u_{n}(x) \frac{t^{n}}{n !} .
$$

It is easily seen that with this definition we have $u_{n}(0)=u_{n}$, and in general

$$
u_{n}(x)=\sum_{k=0}^{n}\left(\begin{array}{l}
n \\
k
\end{array}\right) u_{n-k} x^{k} .
$$

The sequence $u_{n}(x)$ is therefore an example of an Appell family of polynomials, since the degree of $u_{n}$ is $n$ and $\frac{d}{d x} u_{n}(x)=n u_{n-1}(x)$ for all $n$. Furthermore, since

$$
\psi\left(T^{p \tau} h(T)\right)=T^{\tau}(\psi h)(T)
$$

for $\tau \in \mathbb{Z}_{p}$, we have

$$
\left(a_{n}(p \tau)\right)^{*}=a_{n}^{*}(\tau)
$$

or equivalently,

$$
\widehat{a_{n}(p \tau)}=a_{n}(p \tau)-p^{n} a_{n}^{*}(\tau),
$$

for $\tau \in \mathbb{Z}_{p}$. These considerations demonstrate that congruences associated to a sequence $\left\{a_{n}\right\}$ produced by Theorem 2.1 extend immediately to $\left\{a_{n}(x)\right\}$ for $x \in p \mathbb{Z}_{p}$, as we record below:

TheOREM 2.2. Let $h \in \mathfrak{O}_{K}[[T-1]]$ and write $\varphi h\left(e^{t}\right)=\sum_{n=0}^{\infty} \widehat{a}_{n} t^{n} / n$ !. Then $\widehat{a}_{n}(x) \in \mathfrak{O}_{K}[x]$ for all $n$. Furthermore, if $c \equiv 0\left(\bmod \phi(q) p^{a}\right)$ with $a \geq 0$ then for all $\tau \in \mathbb{Z}_{p}$,

$$
\Delta_{c}^{k}\left\{\widehat{a_{m}(p \tau)}\right\} \equiv 0\left(\bmod p^{k a^{+}} \mathfrak{O}_{K}\right)
$$

for all $m, k \geq 0$, and

$$
\left(\begin{array}{c}
p^{-r} \Delta_{c} \\
k
\end{array}\right)\left\{a_{m}(p \tau)\right\} \in \mathfrak{O}_{K}
$$

for $0 \leq r \leq a^{+}$and all $m, k \geq 0$, where $\widehat{a_{m}(p \tau)}$ is as in (2.17). 
3. Congruences for Bernoulli and Euler polynomials. Recall that Dwork's shift map $x \mapsto x^{\prime}$ is defined for $x \in \mathbb{Z}_{p}$ by the relation $p x^{\prime}-x=$ $\mu_{x} \in\{0,1, \ldots, p-1\}$, so that $\mu_{x}$ is the representative of $-x \bmod p \mathbb{Z}_{p}$ which lies in $\{0,1, \ldots, p-1\}$. The following lemma describes the action of Dwork's $\psi$ operator on certain functions in terms of the shift map.

Lemma 3.1. For $x \in \mathbb{Z}_{p}$ and $(b, p)=1$, we have formally

$$
\psi\left(\frac{T^{b x}}{T^{b}-c}\right)=c^{p-1-\mu_{x}} \frac{T^{b x^{\prime}}}{T^{b}-c^{p}},
$$

or equivalently,

$$
\varphi\left(\frac{T^{b x}}{T^{b}-c}\right)=\frac{T^{b x}}{T^{b}-c}-c^{p-1-\mu_{x}} \frac{T^{b p x^{\prime}}}{T^{b p}-c^{p}} .
$$

Proof. Using (2.4) we compute

$$
\begin{aligned}
(1-\varphi)\left(\frac{T^{x}}{T-c}\right) & =\frac{1}{p} \sum_{\zeta^{p}=1} \frac{\zeta^{x} T^{x}}{\zeta T-c}=T^{x}\left(\frac{1}{p} \sum_{\zeta^{p}=1} \frac{\zeta^{-\mu_{x}}}{\zeta T-c}\right) \\
& =T^{x}\left(c^{p-1-\mu_{x}} \frac{T^{\mu_{x}}}{T^{p}-c^{p}}\right)
\end{aligned}
$$

by considering the partial fraction decomposition of the latter rational function. The result in the case $b=1$ follows immediately by noting that $x+\mu_{x}=$ $p x^{\prime}$. To get the result for general $b$, note that $\varphi\left(h\left(T^{b}\right)\right)=(\varphi h)\left(T^{b}\right)$ for $(b, p)=1$, since in this case the $\operatorname{map} \zeta \mapsto \zeta^{b}$ permutes the solutions of $\zeta^{p}=1$.

Theorem 3.2. Suppose $\phi(q)$ does not divide $m$. If $c \equiv 0\left(\bmod \phi(q) p^{a}\right)$ with $a \geq 0$, then for all $x \in \mathbb{Z}_{p}$,

and

$$
\Delta_{c}^{k}\left\{\frac{B_{m}(x)-p^{m-1} B_{m}\left(x^{\prime}\right)}{m}\right\} \equiv 0\left(\bmod \frac{1}{2} p^{k a^{+}} \mathbb{Z}_{p}\right)
$$

$$
\left(\begin{array}{c}
p^{-r} \Delta_{c} \\
k
\end{array}\right)\left\{\frac{B_{m}(x)-p^{m-1} B_{m}\left(x^{\prime}\right)}{m}\right\} \in \frac{1}{2} \mathbb{Z}_{p}
$$

for $0 \leq r \leq a^{+}$and all $k>0$.

Proof. Let $b$ be any positive integer with $(b, p)=1$, and define

$$
h(T)=\frac{b T^{b x}}{T^{b}-1}-\frac{T^{x}}{T-1} .
$$

By writing

$$
h(T)=\frac{1}{T-1}\left(\frac{b T^{b x}}{\Phi_{b}(T)}-T^{x}\right)
$$

with $\Phi_{b}(T)=T^{b-1}+T^{b-2}+\ldots+T+1$ and observing that the latter factor in (3.3) lies in $\mathbb{Z}_{p}[[T-1]]$ and has constant term zero, we see that 
$h \in \mathbb{Z}_{p}[[T-1]]$. Applying Lemma 3.1 gives

$$
(1-\varphi) h(T)=\frac{b T^{b p x^{\prime}}}{T^{b p}-1}-\frac{T^{p x^{\prime}}}{T^{p}-1},
$$

and substitution of $T=e^{t}$ then yields

$$
\varphi h\left(e^{t}\right)=\sum_{n=1}^{\infty}\left(b^{n}-1\right)\left(\frac{B_{n}(x)-p^{n-1} B_{n}\left(x^{\prime}\right)}{n}\right) \frac{t^{n-1}}{(n-1) !} .
$$

So corresponding to the function $h(T)$ in (3.2), the congruences of Theorem 2.1 hold for the numbers

$$
\widehat{a}_{n}=\left(b^{n+1}-1\right) \frac{B_{n+1}(x)-p^{n} B_{n+1}\left(x^{\prime}\right)}{n+1} .
$$

Now supposing that $n+1$ is not divisible by $\phi(q)$, choose $b$ so that $b^{n+1} \not \equiv 1(\bmod q)$. Then since the congruences of Theorem 2.1 hold for the numbers $\widehat{a}_{n}$ in (3.6) associated to $(-1)^{p-1} b^{p^{i}}$ for $i=1,2, \ldots$, they hold for the numbers

$$
\left(\omega(b)^{n+1}-1\right) \frac{B_{n+1}(x)-p^{n} B_{n+1}\left(x^{\prime}\right)}{n+1}
$$

obtained upon passing to the $p$-adic limit (because $(-1)^{p-1} b^{p^{i}} \rightarrow \omega(b)$ in $\left.\mathbb{Z}_{p}\right)$. Put $m=n+1$. Since $\omega(b)^{m+j c}=\omega(b)^{m}$ for all integers $j$, we have

$$
\begin{aligned}
\Delta_{c}^{k}\left\{\left(\omega(b)^{m}-1\right) \frac{B_{m}(x)-p^{m-1} B_{m}\left(x^{\prime}\right)}{m}\right\} & \\
=\left(\omega(b)^{m}-1\right) \Delta_{c}^{k} & \left\{\frac{B_{m}(x)-p^{m-1} B_{m}\left(x^{\prime}\right)}{m}\right\}
\end{aligned}
$$

for all $k$, that is, the constant factor $\omega(b)^{m}-1$ may be removed from each term in the congruences. Finally, noting that $\operatorname{ord}_{p}\left(\omega(b)^{m}-1\right)=\operatorname{ord}_{p} 2$ for all primes $p$ gives the result of the theorem.

The generalized Euler polynomials $H_{n}(u, x)$ attached to an algebraic number $u \neq 1$ have been defined by

$$
\left(\frac{1-u}{e^{t}-u}\right) e^{x t}=\sum_{n=0}^{\infty} H_{n}(u, x) \frac{t^{n}}{n !}
$$

in [9]. For our purposes $u$ will be an algebraic integer for which $1-u$ is a $p$-adic unit. When $p>2$ and $u=-1$ one obtains the usual Euler polynomials $E_{n}(x)=H_{n}(-1, x)$. Theorem 2.1 may be applied to give congruences for $H_{n}(u, x)$ for $x \in \mathbb{Z}_{p}$.

TheOREM 3.3. Let $u$ be algebraic over $\mathbb{Q}_{p}$, and suppose that $1-u \in \mathfrak{O}_{K}^{\times}$, where $K=\mathbb{Q}_{p}(u)$. If $c \equiv 0\left(\bmod \phi(q) p^{a}\right)$ then for all $x \in \mathbb{Z}_{p}$ we have 


$$
\Delta_{c}^{k}\left\{H_{m}(u, x)-u^{p-1-\mu_{x}} \frac{1-u}{1-u^{p}} p^{m} H_{m}\left(u^{p}, x^{\prime}\right)\right\} \equiv 0\left(\bmod p^{k a^{+}} \mathfrak{O}_{K}\right)
$$

and

$$
\left(\begin{array}{c}
p^{-r} \Delta_{c} \\
k
\end{array}\right)\left\{H_{m}(u, x)-u^{p-1-\mu_{x}} \frac{1-u}{1-u^{p}} p^{m} H_{m}\left(u^{p}, x^{\prime}\right)\right\} \in \mathfrak{O}_{K}
$$

for $0 \leq r \leq a^{+}$and all $k>0$.

Proof. If $1-u \in \mathfrak{O}_{K}^{\times}$then $h(T)=((1-u) /(T-u)) \cdot T^{x} \in \mathfrak{O}_{K}[[T-1]]$ for all $x \in \mathbb{Z}_{p}$. From Lemma 3.1 we have

$$
(1-\varphi) h(T)=u^{p-1-\mu_{x}}(1-u) \frac{T^{p x^{\prime}}}{T^{p}-u^{p}} .
$$

Setting $T=e^{t}$ and expanding $\varphi h\left(e^{t}\right)=\sum_{n} \widehat{a}_{n} t^{n} / n$ ! as formal power series gives

$$
\widehat{a}_{m}=H_{m}(u, x)-u^{p-1-\mu_{x}} \frac{1-u}{1-u^{p}} p^{m} H_{m}\left(u^{p}, x^{\prime}\right) .
$$

The theorem then follows from Theorem 2.1.

In many applications of Euler polynomials the parameter $u$ is taken to be a nontrivial $(p-1)$ st root of unity. We observe that in this case the numbers $\widehat{a}_{m}$ in the congruences simplify to

$$
\widehat{a}_{m}=H_{m}(u, x)-u^{-\mu_{x}} p^{m} H_{m}\left(u, x^{\prime}\right) .
$$

If in addition $x$ is a rational number in $[0,1]$ with denominator dividing $p-1$ then $x^{\prime}=x$ and we have

$$
\widehat{a}_{m}=\left(1-u^{-\mu_{x}} p^{m}\right) H_{m}(u, x) .
$$

4. Congruences for generalized Bernoulli polynomials. For a primitive Dirichlet character $\chi$ of conductor $f=f_{\chi}$ the generalized Bernoulli polynomials $B_{n, \chi}(x)$ are defined by

$$
\left(\sum_{a=1}^{f} \frac{\chi(a) t e^{a t}}{e^{f t}-1}\right) e^{x t}=\sum_{n=0}^{\infty} B_{n, \chi}(x) \frac{t^{n}}{n !} .
$$

We begin this section by using Theorem 2.2 to produce congruences for $B_{n, \chi}(x)$ for $x \in p \mathbb{Z}_{p}$ and characters $\chi$ whose conductor is not a power of $p$. All the congruences of this section are extensions of congruences of the type given for $x=0$ which may be found in [2], [5], and [8].

TheOREM 4.1. Suppose that $\chi$ is a primitive Dirichlet character whose conductor $f$ is not a power of $p$, and put $K=\mathbb{Q}_{p}(\chi)$. If $c \equiv 0\left(\bmod \phi(q) p^{a}\right)$ with $a \geq 0$, then for all $\tau \in \mathbb{Z}_{p}$,

$$
\Delta_{c}^{k}\left\{\frac{B_{m, \chi}(p \tau)-\chi(p) p^{m-1} B_{m, \chi}(\tau)}{m}\right\} \equiv 0\left(\bmod p^{k a^{+}} \mathfrak{O}_{K}\right)
$$


and

$$
\left(\begin{array}{c}
p^{-r} \Delta_{c} \\
k
\end{array}\right)\left\{\frac{B_{m, \chi}(p \tau)-\chi(p) p^{m-1} B_{m, \chi}(\tau)}{m}\right\} \in \mathfrak{O}_{K}
$$

for $0 \leq r \leq a^{+}$and all $m, k>0$.

Proof. Since $f$ is not a power of $p$, we may write $f$ in the form $f=d p^{e}$ with $e \geq 0,(d, p)=1$, and $d \neq 1$. Define the rational function

$$
h_{\chi}(T)=\sum_{a=1}^{f} \frac{\chi(a) T^{a}}{T^{f}-1} .
$$

We claim that $h_{\chi} \in \mathfrak{O}_{K}[[T-1]]$. To show this, observe that if $\zeta^{p^{e}}=1$, then $\sum_{a=1}^{f} \chi(a) \zeta^{a}=0$ by ([10], Lemma 4.7). Therefore $T^{p^{e}}-1$ divides $\sum_{a=1}^{f} \chi(a) T^{a}$ in $\mathfrak{O}_{K}[T-1]$. We also have

$$
T^{f}-1=\left(T^{p^{e}}-1\right)\left(T^{(d-1) p^{e}}+T^{(d-2) p^{e}}+\ldots+T^{p^{e}}+1\right)
$$

in $\mathfrak{O}_{K}[T-1]$, and the latter factor is a unit in $\mathfrak{O}_{K}[[T-1]]$ since its constant term is $d \in \mathfrak{O}_{K}^{\times}$. By dividing both numerator and denominator of (4.2) by $T^{p^{e}}-1$ we may then write $h_{\chi}(T)=g(T) / k(T)$ with $g, k$ elements of $\mathfrak{O}_{K}[T-1]$ and $k$ invertible in $\mathfrak{O}_{K}[[T-1]]$. This proves that $h_{\chi} \in \mathfrak{O}_{K}[[T-1]]$.

We now verify that $(1-\varphi) h_{\chi}(T)=\chi(p) h_{\chi}\left(T^{p}\right)$ as rational functions, by computing

$$
\begin{aligned}
(1-\varphi) h_{\chi}(T) & =\frac{1}{p} \sum_{\zeta^{p}=1}\left(\sum_{a=1}^{f} \frac{\chi(a)(\zeta T)^{a}}{(\zeta T)^{f}-1}\right) \\
& =\frac{1}{p} \sum_{\zeta^{p}=1}\left(\sum_{a=1}^{f p} \frac{\chi(a)(\zeta T)^{a}}{(\zeta T)^{f p}-1}\right) \\
& =\sum_{\substack{a=1 \\
p \mid a}}^{f p} \frac{\chi(a) T^{a}}{T^{f p}-1}=\sum_{b=1}^{f} \frac{\chi(p b) T^{p b}}{T^{f p}-1}=\chi(p) h_{\chi}\left(T^{p}\right),
\end{aligned}
$$

the second equality being obtained by multiplying each numerator and denominator by $1+(\zeta T)^{f}+(\zeta T)^{2 f}+\ldots+(\zeta T)^{(p-1) f}$. Therefore if $h_{\chi}\left(e^{t}\right)=$ $\sum a_{n} t^{n} / n$ ! we have $a_{n}=B_{n+1, \chi} /(n+1)$ and $a_{n}^{*}=\chi(p) B_{n+1, \chi} /(n+1)$. The theorem then follows by taking $m=n+1$ and applying Theorem 2.2.

We say that $\chi$ is a character of the first kind if either $f=d$ or $f=d q$ with $(d, p)=1$; we say that $\chi$ is a character of the second kind if either $f=1$ or $f=q p^{e}$ with $e \geq 1$. If $\chi$ is a primitive Dirichlet character and $n$ is an integer, the symbol $\chi_{n}$ will denote the character $\chi \omega^{-n}$, where $\omega$ is the Teichmüller character. Here we use Theorem 2.1 to produce congruences for $B_{n, \chi_{n}}(x)$ for $x \in p \mathbb{Z}_{p}$ and characters $\chi$ which are not of the second kind. 
TheOREM 4.2. Suppose that $\chi$ is a primitive Dirichlet character which is not of the second kind, and put $K=\mathbb{Q}_{p}(\chi)$. If $c \equiv 0\left(\bmod \phi(q) p^{a}\right)$ with $a \geq 0$, then for all $\tau \in \mathbb{Z}_{p}$,

$$
\Delta_{c}^{k}\left\{\frac{B_{m, \chi_{m}}(p \tau)-\chi_{m}(p) p^{m-1} B_{m, \chi_{m}}(\tau)}{m}\right\} \equiv 0\left(\bmod \frac{1}{2} p^{k a^{+}} \mathfrak{O}_{K}\right)
$$

and

$$
\left(\begin{array}{c}
p^{-r} \Delta_{c} \\
k
\end{array}\right)\left\{\frac{B_{m, \chi_{m}}(p \tau)-\chi_{m}(p) p^{m-1} B_{m, \chi_{m}}(\tau)}{m}\right\} \in \frac{1}{2} \mathfrak{O}_{K}
$$

for $0 \leq r \leq a^{+}$and all $m, k>0$.

Proof. First assume that the conductor $f=f_{\chi}$ of $\chi$ is not a power of $p$. Then the conductor $f=f_{\chi_{m}}$ of $\chi_{m}$ is also not a power of $p$ for any $m$. Furthermore, $\omega^{c}=1$, so $\chi_{m+j c}=\chi_{m}$ for all $j$. The theorem in this case follows immediately from Theorem 4.1.

We have now reduced to the case $f_{\chi}=q$, so that $\chi$ is a power of the Teichmüller character $\omega$. In this case $f_{\chi_{m}}$ is either $q$ or 1 , and if $f_{\chi_{m}}=1$, then $\phi(q)$ does not divide $m$, since $\chi$ is nontrivial. So in the case where $f_{\chi}=q, f_{\chi_{m}}=1$, the theorem follows from Theorem 3.2 by observing that $\chi_{m+j c}=\chi_{m}=1$ for all $j$ and using the identity $B_{n, 1}(x)=(-1)^{n} B_{n}(-x)$.

The remaining case is $f=f_{\chi}=f_{\chi_{m}}=q$. Let $n$ be any positive integer with $f_{\chi_{n}}=q$, let $b$ be a positive integer with $(b, p)=1$, and define

$$
h_{\chi_{n}}(T)=b \chi_{n}(b) T^{b x}\left(\sum_{a=1}^{f} \frac{\chi_{n}(a) T^{a b}}{T^{b f}-1}\right)-T^{x} \sum_{a=1}^{f} \frac{\chi_{n}(a) T^{a}}{T^{f}-1},
$$

where $x \in p \mathbb{Z}_{p}$. Equivalently we may write

$$
\begin{aligned}
h_{\chi_{n}}(T) & =\frac{b \chi_{n}(b) T^{b x}\left(\sum_{a=1}^{f} \chi_{n}(a) T^{a b}\right)-T^{x} \sum_{a=1}^{f b} \chi_{n}(a) T^{a}}{T^{b f}-1} \\
& =\frac{g(T)}{T^{b f}-1}
\end{aligned}
$$

with $g(T) \in \mathfrak{O}_{K}[[T-1]]$. If $\zeta^{q}=1$, then

$$
\begin{aligned}
g(\zeta) & =b \chi_{n}(b) \zeta^{b x}\left(\sum_{a=1}^{f} \chi_{n}(a) \zeta^{a b}\right)-\zeta^{x} \sum_{a=1}^{f b} \chi_{n}(a) \zeta^{a} \\
& =b\left(\zeta^{b x} \sum_{a=1}^{f} \chi_{n}(a b) \zeta^{a b}-\zeta^{x} \sum_{a=1}^{f} \chi_{n}(a) \zeta^{a}\right) \\
& =b\left(\zeta^{b x}-\zeta^{x}\right)\left(\sum_{a=1}^{f} \chi_{n}(a) \zeta^{a}\right)=0,
\end{aligned}
$$


since $(b-1) x \equiv 0\left(\bmod q \mathbb{Z}_{p}\right)$ for $x \in p \mathbb{Z}_{p}$ and $(b, p)=1$. This shows that $T^{f}-1$ divides $g(T)$ in $\mathfrak{O}_{K}[[T-1]]$. Since $T^{b f}-1$ is also divisible by $T^{f}-1$ in $\mathfrak{O}_{K}[T-1]$ and the quotient is a unit in $\mathfrak{O}_{K}[[T-1]]^{\times}$, we see that $h_{\chi_{n}} \in$ $\mathfrak{O}_{K}[[T-1]]$.

We now obtain $a_{m-1}=\left(b^{m} \chi_{n}(b)-1\right) B_{m, \chi_{n}}(x) / m$ by using equation (4.1) to expand $h_{\chi_{n}}\left(e^{t}\right)=\sum a_{m} t^{m} / m$ !. Furthermore, as in (4.4) we can compute

$$
(1-\varphi) h_{\chi_{n}}(T)=b \chi_{n}(b)\left(\sum_{\substack{a=1 \\ p \mid a}}^{f} \frac{\chi_{n}(a) T^{b(a+x)}}{T^{b f}-1}\right)-\sum_{\substack{a=1 \\ p \mid a}}^{f} \frac{\chi_{n}(a) T^{a+x}}{T^{f}-1}=0
$$

since $\chi_{n}(a)=0$ if $p$ divides $a$; therefore $\widehat{a}_{m}=a_{m}$ for all $m$.

Since $\chi$ is nontrivial, we may choose a value of $b$ for which $(b, p)=1$ and $\chi(b) \neq 1$. The congruences of Theorem 2.1 hold for the numbers $\widehat{a}_{m-1}$ associated to $b$ and to $(-1)^{p-1} b^{p^{i}}$ for $i=1,2, \ldots$, so they also hold for the numbers

$$
\left(\omega(b)^{m} \chi_{n}(b)-1\right) B_{m, \chi_{n}}(x) / m
$$

obtained upon passing to the $p$-adic limit. Since $\omega^{m}=\omega^{m+j c}$ for all $j$, the constant factor $\omega(b)^{m} \chi_{n}(b)-1$ may be factored out of each term in the congruences. Now set $n=m$. Since $\chi(b)$ and $\omega(b)$ are both nonzero, $\omega(b)^{m} \chi_{m}(b)-1$ equals $\chi(b)-1$. Observing that $\operatorname{ord}_{p}(\chi(b)-1)=\operatorname{ord}_{p} 2$ for all primes $p$, putting $x=p \tau$, and observing that $\chi_{m}(p)=0$ completes the proof.

In [6] G. Fox studied a two-variable $p$-adic $L$ function $L_{p}(s, \tau, \chi)$ which is defined for $s, \tau$ lying in the $p$-adic completion $\mathbb{C}_{p}$ of an algebraic closure of $\mathbb{Q}_{p}$ and satisfying $\operatorname{ord}_{p} \tau \geq 0$ and $\operatorname{ord}_{p} s \geq 1 /(p-1)-\operatorname{ord}_{p} q$. The members of the congruences in the above theorem are values of this $L$-function at negative integer values of $s$. Specifically, Fox showed that if $n$ is a positive integer then

$$
L_{p}(1-n, \tau, \chi)=\frac{1}{n}\left(B_{n, \chi_{n}}(p \tau)-\chi_{n}(p) p^{n-1} B_{n, \chi_{n}}(\tau)\right) .
$$

In both (4.10) and Theorem 4.2 the main term Bernoulli polynomial has an argument whose $p$-adic ordinal must be at least 1 . We conclude with an analogue of Theorem 3.2 for values of generalized Bernoulli polynomials at an argument which is not restricted to lie in $p \mathbb{Z}_{p}$, extending Theorem 4.1. Unfortunately this requires certain impositions upon the conductor of $\chi$. For this result we make use of the decomposition of $\chi$ as $\chi=\chi_{(0)} \chi_{(p)}$, where the conductor $f_{0}$ of $\chi_{(0)}$ is relatively prime to $p$ and the conductor $f_{p}$ of $\chi_{(p)}$ is a power of $p$ (cf. [10], p. 23). 
TheOREM 4.3. Suppose that $\chi$ is a primitive Dirichlet character such that $f_{0} \neq 1$ and $f_{p}=1$ or $p$, and put $K=\mathbb{Q}_{p}(\chi)$. Let $x \in \mathbb{Z}_{p}$ be such that $f_{0}$ divides $\mu_{x}$ in $\mathbb{Z}$. If $c \equiv 0\left(\bmod \phi(q) p^{a}\right)$ with $a \geq 0$, then

$$
\Delta_{c}^{k}\left\{\frac{B_{m, \chi}(x)-\chi_{(p)}\left(\mu_{x}\right) \chi_{(0)}(p) p^{m-1} B_{m, \chi_{(0)}}\left(x^{\prime}\right)}{m}\right\} \equiv 0\left(\bmod p^{k a^{+}} \mathfrak{O}_{K}\right)
$$

and

$$
\left(\begin{array}{c}
p^{-r} \Delta_{c} \\
k
\end{array}\right)\left\{\frac{B_{m, \chi}(x)-\chi_{(p)}\left(\mu_{x}\right) \chi_{(0)}(p) p^{m-1} B_{m, \chi_{(0)}}\left(x^{\prime}\right)}{m}\right\} \in \mathfrak{O}_{K}
$$

for $0 \leq r \leq a^{+}$and all $m, k>0$.

Proof. Suppose that $x \in \mathbb{Z}_{p}$ is such that $f_{0}$ divides $\mu_{x}$ in $\mathbb{Z}$. Define the function

$$
h_{\chi}(T, x)=\sum_{a=1}^{f} \frac{\chi(a) T^{a+x}}{T^{f}-1} .
$$

As the product of $T^{x}$ and the function $h_{\chi}(T)$ in (4.2), this function lies in $\mathfrak{O}_{K}[[T-1]]$. We compute

$$
\begin{aligned}
(1-\varphi) h_{\chi}(T, x) & =\frac{1}{p} \sum_{\zeta^{p}=1}\left(\sum_{a=1}^{f} \frac{\chi(a)(\zeta T)^{a+x}}{(\zeta T)^{f}-1}\right) \\
& =\frac{1}{p} \sum_{\zeta^{p}=1}\left(\sum_{a=1}^{f p} \frac{\chi(a)(\zeta T)^{a+x}}{(\zeta T)^{f p}-1}\right)=\sum_{\substack{a=1 \\
p \mid a+x}}^{f p} \frac{\chi(a) T^{a+x}}{T^{f p}-1} .
\end{aligned}
$$

Since $\left(f_{0}, f_{p}\right)=1$, we have $\chi(a)=\chi_{(0)}(a) \chi_{(p)}(a)$ for all $a$. If $p$ divides $a+x$ in $\mathbb{Z}_{p}$ then $a \equiv \mu_{x}(\bmod p)$ and therefore $\chi_{(p)}(a)=\chi_{(p)}\left(\mu_{x}\right)$. Then by writing $p b=a+x$ as $b$ runs from $x^{\prime}$ to $x^{\prime}+f-1$, equation (4.12) becomes

$$
\begin{aligned}
(1-\varphi) h_{\chi}(T, x) & =\chi_{(p)}\left(\mu_{x}\right) \sum_{\substack{a=1 \\
p \mid a+x}}^{f p} \frac{\chi_{(0)}(a) T^{a+x}}{T^{f p}-1} \\
& =\chi_{(p)}\left(\mu_{x}\right) \sum_{c=0}^{f-1} \frac{\chi_{(0)}\left(\mu_{x}+p c\right) T^{p\left(c+x^{\prime}\right)}}{T^{f p}-1} \\
& \left.=\chi_{(p)}\left(\mu_{x}\right) \sum_{c=0}^{f-1} \frac{\chi_{(0)}(p c) T^{p\left(c+x^{\prime}\right)}}{T^{f p}-1} \quad \text { (since } f_{0} \mid \mu_{x}\right) \\
& =\chi_{(p)}\left(\mu_{x}\right) \chi_{(0)}(p) h_{\chi_{(0)}}\left(T^{p}, x^{\prime}\right) .
\end{aligned}
$$

Therefore if $h_{\chi}\left(e^{t}, x\right)=\sum a_{n} t^{n} / n$ ! we have $a_{n}=B_{n+1, \chi}(x) /(n+1)$ for all $n$ and $a_{n}^{*}=\chi_{(p)}\left(\mu_{x}\right) \chi_{(0)}(p) B_{n+1, \chi_{(0)}}\left(x^{\prime}\right) /(n+1)$. The theorem then follows by taking $m=n+1$ and applying Theorem 2.1. 
By using the identity $B_{n, \chi}(-x)=(-1)^{n} \chi(-1) B_{n, \chi}(x)$ and the fact that $(-x)^{\prime}=1-x^{\prime}$ and $\mu_{-x}=p-\mu_{x}$ when $\mu_{x} \neq 0$ we obtain the following variation of Theorem 4.3.

COROllary 4.4. Suppose that $\chi$ is a primitive Dirichlet character such that $f_{0} \neq 1$ and $f_{p}=1$ or $p$, and put $K=\mathbb{Q}_{p}(\chi)$. Let $x \in \mathbb{Z}_{p}$ be such that $f_{0}$ divides $p-\mu_{x}$ in $\mathbb{Z}$. If $c \equiv 0\left(\bmod \phi(q) p^{a}\right)$ with $a \geq 0$, then

$$
\Delta_{c}^{k}\left\{\frac{B_{m, \chi}(x)-\chi_{(p)}\left(\mu_{x}\right) \chi_{(0)}(p) p^{m-1} B_{m, \chi_{(0)}}\left(x^{\prime}-1\right)}{m}\right\} \equiv 0\left(\bmod p^{k a^{+}} \mathfrak{O}_{K}\right)
$$

and

$$
\left(\begin{array}{c}
p^{-r} \Delta_{c} \\
k
\end{array}\right)\left\{\frac{B_{m, \chi}(x)-\chi_{(p)}\left(\mu_{x}\right) \chi_{(0)}(p) p^{m-1} B_{m, \chi_{(0)}}\left(x^{\prime}-1\right)}{m}\right\} \in \mathfrak{O}_{K}
$$

for $0 \leq r \leq a^{+}$and all $m, k>0$.

\section{References}

[1] L. Carlitz, Some congruences for the Bernoulli numbers, Amer. J. Math. 75 (1953), 163-172.

[2] - Arithmetic properties of generalized Bernoulli numbers, J. Reine Angew. Math. 202 (1959), 174-182.

[3] B. Dwork, Lectures on p-adic Differential Equations, Springer, New York, 1982.

[4] M. Eie and Y. L. Ong, A generalization of Kummer's congruence, Abh. Math. Sem. Univ. Hamburg 67 (1997), 149-157.

[5] R. Ernvall, Generalized Bernoulli numbers, generalized irregular primes, and class number, Ann. Univ. Turku. Ser. A I 178 (1979), 72 pp.

[6] G. J. Fox, A p-adic L-function of two variables, Enseign. Math. 46 (2000), 225-278.

[7] H. S. Gunaratne, A new generalisation of the Kummer congruence, in: Computational Algebra and Number Theory, Math. Appl. 325, Kluwer, Dordrecht, 1995, $255-265$.

[8] K. Shiratani, Kummer's congruence for generalized Bernoulli numbers and its application, Mem. Fac. Sci. Kyushu Univ. Ser. A 26 (1971), 119-138.

[9] H. Tsumura, On a p-adic interpolation of the generalized Euler numbers and its applications, Tokyo J. Math. 10 (1987), 281-293.

[10] L. Washington, Introduction to Cyclotomic Fields, Springer, New York, 1982.

[11] P. T. Young, Congruences for Bernoulli, Euler, and Stirling numbers, J. Number Theory 78 (1999), 204-227.

Department of Mathematics

University of Charleston

Charleston, SC 29424, U.S.A.

E-mail:paul@math.cofc.edu 